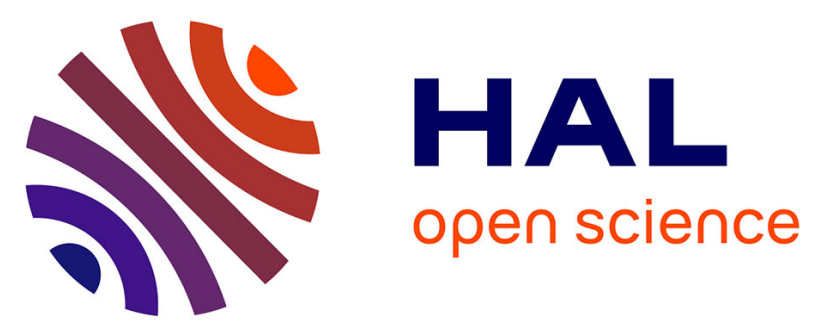

\title{
Entrance and exit effects for a viscoelastic liquid displacing a simple liquid through a contraction.
}

J. Avendano, Nicolas Pannacci, Benjamin Herzhaft, Patrick Gateau, Philippe

Coussot

\section{- To cite this version:}

J. Avendano, Nicolas Pannacci, Benjamin Herzhaft, Patrick Gateau, Philippe Coussot. Entrance and exit effects for a viscoelastic liquid displacing a simple liquid through a contraction.. Journal of Non-Newtonian Fluid Mechanics, 2013, 199 (September), pp.51-60. 10.1016/j.jnnfm.2013.06.005 . hal-01071704

\section{HAL Id: hal-01071704 \\ https://hal-ifp.archives-ouvertes.fr/hal-01071704}

Submitted on 6 Oct 2014

HAL is a multi-disciplinary open access archive for the deposit and dissemination of scientific research documents, whether they are published or not. The documents may come from teaching and research institutions in France or abroad, or from public or private research centers.
L'archive ouverte pluridisciplinaire HAL, est destinée au dépôt et à la diffusion de documents scientifiques de niveau recherche, publiés ou non, émanant des établissements d'enseignement et de recherche français ou étrangers, des laboratoires publics ou privés. 


\title{
Entrance and exit effects for a viscoelastic liquid displacing a simple liquid through a contraction
}

\author{
J. Avendano 1,", N. Pannacci ${ }^{1}$, B. Herzhaft ${ }^{1}$, P. Gateau ${ }^{1}$, P. Coussot ${ }^{2}$ \\ ${ }^{\prime}$ IFP Energies nowvelles, 1 et 4 avenue de Bois-Préau, 92852 Rueil-Malmaison, France \\ ${ }^{2}$ Université Paris-Est, Laboratoire Navier, Champs sur Marne, France \\ "Now with Universidad de los Andes, Laboratorio FIRP, Mérida, Venezuela
}

\begin{abstract}
We studied the displacement of the interface between a viscoelastic fluid pushing a simple liquid through a rectangular contraction by following the front interface deformation in time. The progressive deformation of the interface until apparent stabilization is followed, which makes it possible to identify a transient and a stationary regime. For low Weissenberg number the shape of the interface is essentially similar to that between two simple liquids. For sufficiently large Weissenberg number the shape of the interface is different: it is narrower before the entrance and wider just after the exit. The characteristics of this shape are qualitatively analogous to those of the interface between the vortices and the convected regions for the flow of a single viscoelastic fluid through a contractionexpansion. This suggests that the entrance effect is due to extensional effects and the exit effect is due to normal stress effects.
\end{abstract}

\section{Introduction}

Chemical Enhanced Oil recovery processes consist in the injection of a chemical formulation into the reservoir that will promote the displacement of oil toward the producing well. The polymers commonly used in polymer flooding are synthetic polyacrylamides at concentrations ranging from 200 to $2000 \mathrm{ppm}$, with high molecular weight - usually around $2010^{6} \mathrm{~g} \cdot \mathrm{mol}^{-1}$ - and with various degrees of hydrolysation [1]. The basic expected effect is the increase of the viscosity of the displacing fluid beyond that of the displaced fluid, which allows a stable oil displacement and therefore improves the rate of oil recovery as compared to a simple water flooding. Indeed the displacement of a fluid by a more viscous one is a priori not affected by the Saffman-Taylor instability [2]. However, even if using a displacing fluid more viscous than the displaced fluid tends to stabilize the injection front at a macroscopic scale allowing a better sweeping of the reservoir, at a local scale, i.e. at the pore scale, the residual oil saturation (i.e. the oil remaining in the porous rock after flooding) is not modified compared to simple water flooding. However, some recent publications suggested that the residual oil saturation could be modified by the elastic character of the solutions [3-5]

The flow of a Newtonian fluid displacing another Newtonian fluid through a porous medium has been widely studied in relation with oil recovery [6-8]. It was shown that depending on the viscosity ratio between the fluids and the value of the capillary number $(\mathrm{Ca})$, three different regimes occur: viscous fingering (Saffman Taylor instability), capillary digitation (when the capillary forces drive the instability) 
and stable flow. In the case of a viscoelastic fluid displaced under unstable conditions the finger width appears to vary with the elastic character of the fluid $[9,10]$.

As far as we know the flow of a viscoelastic fluid displacing another (less viscous) fluid through a porous medium has never been studied in a fundamental way. For such two phase flows we can expect some new effects since for monophasic flow of viscoelastic fluid in complex geometry several specific effects are known to occur. Indeed for example some additional pressure term can exist in dead-end pore with fluids exhibiting significant normal stresses [11]. It is also well-known that a vortex can form in the corners before the entrance through an abrupt contraction $[12,13]$. The size of this vortex was in particular shown to increase with the elastic character, fluid velocity and contraction ratio [14-17]. In such a situation elongational effects play an important role [18-19]. Besides vortices were observed in the corners just after the entrance flow in an expansion: the size (length) of these vortices was shown to increase with the Reynolds number for a Newtonian fluid; for viscoelastic fluids it was shown to slightly decrease for an increasing Weissenberg (or Deborah) number through a square-square expansion [sousa 2011] [2022] but simulations suggest this vortex stabilizes at sufficiently high Weissenberg numbers [21]. At last we have the well-known effect of expansion of a jet of viscoelastic fluid in the open air in which normal stress effects play a major role [23]. It was also suggested that normal stress effects play a significant role in the decrease of the entrance correction below the Newtonian value [13].

A recent work [24] specifically focused on the flow of viscoelastic fluids through more complex geometries approaching the complexity of a real porous medium. Through a detailed study of the flow properties and fluid behavior the authors showed that the velocity profiles become progressively asymmetric when the Deborah number increases. They also showed that the resistance law departs from the expected one for a Newtonian fluid when the Deborah number is sufficiently large. It was suggested that normal stress effects can play a major role in this phenomenon. Recently the shape of the interface between a viscoelastic fluid pushing a Newtonian fluid moving along a straight channel was studied [25]. It was shown that when normal stress effects are sufficiently large the viscoelastic fluid tends to occupy a wider area during its penetration through the simple liquid.

In order to focus on the specific impact at a local scale of a viscoelastic fluid pushing a simple liquid through a porous medium we carried out tests in a very simple pore geometry, i.e. a thin rectangular channel with a short restriction of its width at some place, and we observed the deformation of the interface between the two fluids during the displacement through the restriction. We studied the impact of the fluid velocity on the evolution of the interface, with a Newtonian fluid and progressively more concentrated polymer solutions (Boger fluids) [26-27] as displacing fluids. Materials and methods are 
described first (Section 2), then we present the experimental results (Section 3). In Section 4 we analyze and discuss the trends observed.

\section{Materials and procedures}

\subsection{Materials}

The displaced fluid is a Newtonian Silicone oil: Rhodorsil HV-100, of viscosity equal to 0.1 Pa.s. All displacing fluids were immiscible with the displaced fluid. Two families of viscoelastic displacing fluids were formulated. The first one is an aqueous solution containing a non-ionic Polyacrylamide (Aldrich, 4000 or $\left.6000 \mathrm{~kg} \cdot \mathrm{mol}^{-1}\right)\left(0.6 \%\right.$ in weight) and a commercial Newtonian cane syrup (Canadou ${ }^{\circledR}$, called CAN from here) $\left(80 \%\right.$ in weight of sugar) of viscosity equal to $0,16 \mathrm{~Pa} . \mathrm{s}$, with a bactericide $\left(\mathrm{NaN}_{3}\right)$ $(0.05 \%$ in weight). Sodium chloride $(0.2 \%$ in weight) was added to damp possible electrostatic interactions [28] and was found to enhance the reproducibility of data; sodium nitrite was also added and served as a bactericide [29]. The second family is an aqueous solution containing Polyethylene oxide (Aldrich, Molecular Weight: $4000 \mathrm{~kg} \cdot \mathrm{mol}^{-1}$ ) at different concentrations and Polyethylene glycol (Aldrich, $600 \mathrm{~g} / \mathrm{mol})(50 \%$ in weight). All the fluids were prepared by dissolving the high molecular weight polymer in water (primary solvent), then mixing using a magnetic stirrer at $250 \mathrm{rpm}$ for several hours. Then the secondary solvent (depending on the fluid) was added progressively while mixing with a magnetic stirrer at $400 \mathrm{rpm}$ during 4 hours. In the rest of this paper we will call these materials respectively PAM4000, PAM6000, and PEGPEO followed by the high molecular weight polymer concentration in ppm.

The interfacial tension between the fluids and the displaced silicone oil was measured with a TK 100 KRUSS tensiometer using the Wilhelmy plate technique. We found $34.5 \mathrm{mN} / \mathrm{m}$ for CAN, $26.1 \mathrm{mN} . \mathrm{m}^{-1}$ for the PAM solutions, and $38.2 \mathrm{mN} . \mathrm{m}^{-1}$ for the PEO solutions (with an uncertainty of about $5 \%$ on these measurements).

All the rheometrical tests and the displacement experiments (in the channel) were carried out at room temperature $\left(22 \pm 1^{\circ} \mathrm{C}\right)$.

2.2 Rheological behavior in simple shear 
The rheological characteristics of these fluids in steady state simple shear, i.e. the shear stress $\tau$ and the first normal stress difference $N_{1}$ (that we will call normal stress in the following) as a function of the shear rate $\dot{\gamma}$, were measured with a controlled stress rheometer (AR2000, TA Instruments) equipped with a cone and plate geometry (diameter: $4 \mathrm{~cm}$; angle: $2^{\circ}$ ) which seems to be an appropriate geometry for measuring the first normal stress difference since the shear rate is homogeneous and the angle is small. The normal force was measured via a gauge placed below the bottom plate. From the above data we computed the apparent viscosity: $\eta=\tau / \dot{\gamma}$.

After having set up the sample between the tools, the experimental procedure consists to impose first a high shear rate $\left(2000 \mathrm{~s}^{-1}\right)$ during $30 \mathrm{~s}$. Then the sample is left at rest until reaching a stable nomal stress value (where the normal stress sensor is set to 0 ) which means that the measured normal stress during flow is taken from this value. The time needed for this stabilization can be up to several hours. The above residual normal stress value can be due to capillary effects associated with the curvature of the free surface of the sample. After that we successively impose steps of constant shear rate $(\dot{\gamma})$ of increasing levels from $0,1 \mathrm{~s}^{-1}$ up to a high value which depends on the fluid but is usually $1000 \mathrm{~s}^{-1}$, then decreasing the shear rate back to the initial value. At each step we record the equilibrium shear stress and first normal stress difference. The difference between the data (apparent viscosity and normal stress) for the increasing and the decreasing ramps was negligible, which means that these materials did not exhibit thixotropic behavior. Only the data associated with the decreasing ramp are presented here. They were shown to be reproducible, even after thirty days. The Reynolds number at the periphery was always smaller than 0.5 which means that inertia effects were negligible.

In each case it was found that at low shear rates $N_{1}$ fluctuated widely around some value (see for example Figure 1). We considered that these fluctuations result from uncertainties on measurements and/or from artefacts, and we removed the corresponding data. Note that the stress amplitude of these fluctuations is close to the normal stress resolution of the instrument as provided by the supplier for our geometry, i.e. $4 \mathrm{~Pa}$. This does not mean that normal stresses do not exist in that range but that we were able to measure relevant values only at sufficiently high shear rates. 


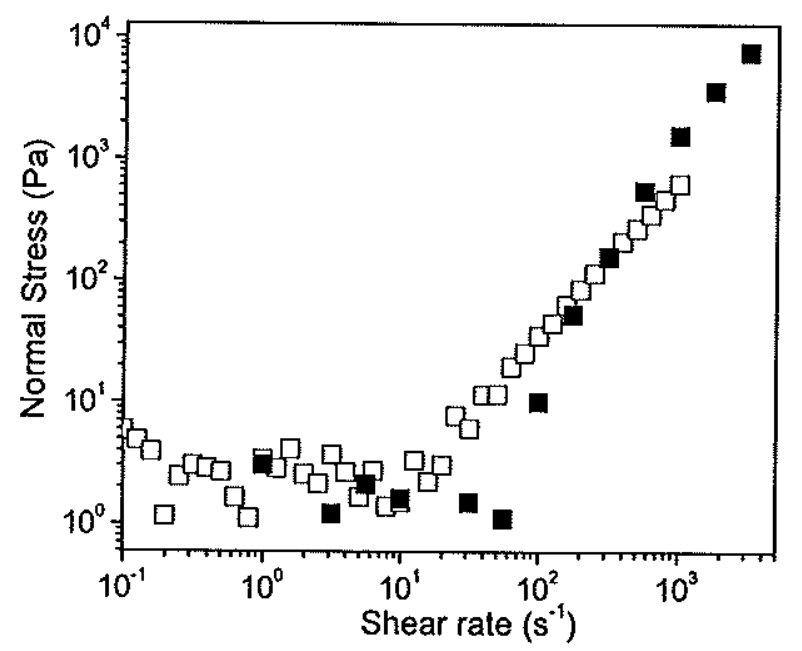

Figure 1: Normal stress data obtained with PAM6000 (open squares) and PEGPEO-2000 (filled squares).

The results of rheological characterization of the different fluid types are shown in Figures 2-3. The viscosity of PAM solutions is almost constant below some critical shear rate then decreases at higher shear rates. This decrease is stronger for higher concentrations. The normal stress, which is larger for higher concentrations, appears to significantly increase precisely in this range of shear-thinning behavior.

The viscosity of PEGPEO can be considered as constant in our range of shear rates. The normal stress is significant approximately in the same range of shear rates as for the PAM solutions, but it increases more slowly with shear rate. The viscosity and normal stress only slightly increase with the polymer concentration in our range of observation. 


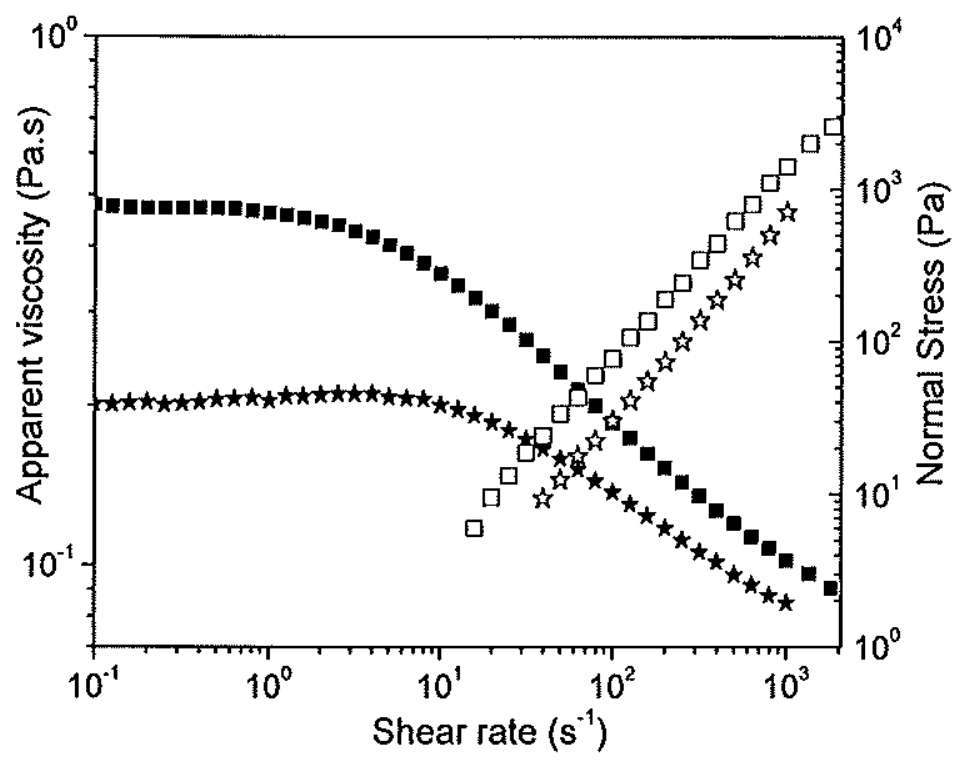

Figure 2: Apparent viscosity (filled symbols) and normal stress (open symbols) as a function of shear rate for PAM4000 (stars) and PAM6000 (squares).

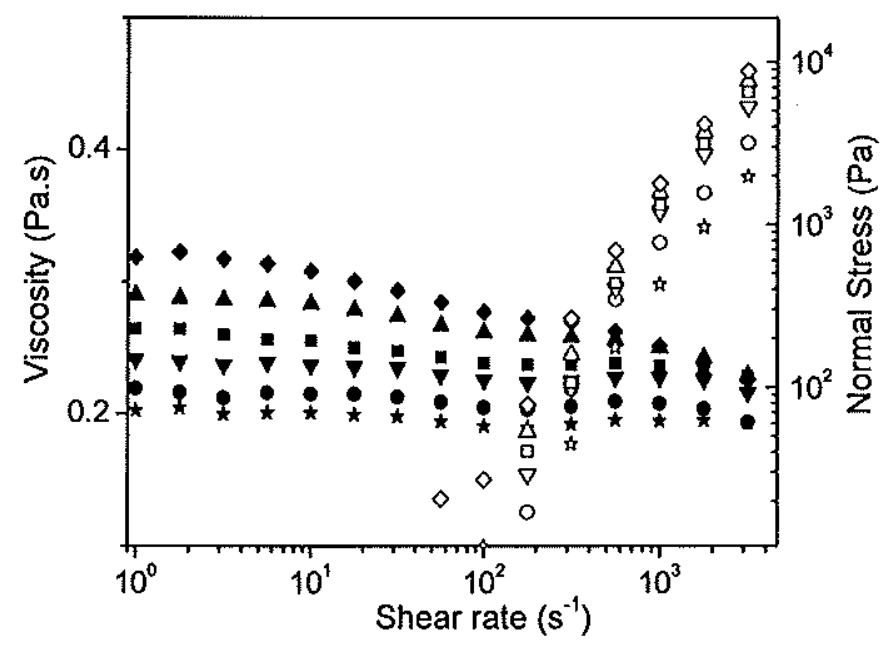

Figure 3: Apparent viscosity (filled symbols) and normal stress (open symbols) as a function of shear rate for PEGPEO at different concentrations (from bottom to top): 250, 500, 1000, 1500, 2000,3000 ppm.

We also carried relaxation tests in order to determine the relaxation time $(\lambda)$ of each of the material used. It appeared that as a first approximation the stress decrease in time could be well fitted by an exponential curve with a single relaxation time. The relaxation times obtained for our materials were: for PAM4000 $0.022 \mathrm{~s}$, for PAM6000 $0.04 \mathrm{~s}$, for PEGPEO $0.018 \mathrm{~s}$ at $500 \mathrm{ppm}, 0.06 \mathrm{~s}$ at $1000 \mathrm{ppm}, 0.12 \mathrm{~s}$ at $2000 \mathrm{ppm}$. 


\subsection{Rheological behavior in extensional flow}

The extensional viscosity $\left(\eta_{E}\right)$ was measured with a VROC rheometer (Rheosense, Inc.), using a technique known as "microfluidic rheometry". This equipment includes a pump to impose a constant flow rate through the flow geometry, which is made of Pyrex and mounted on a silicone coated with gold where the pressure sensors are placed. The chosen geometry, an EVROC chip (planar hyperbolic contraction-expansion) has a thickness $(d)$ much smaller than the width at the entrance $\left(w_{0}\right)$. The width along the contraction $\mathrm{w}(x)$ is described by the relation $w(x)=c / x$. The dimensions of the EVROC chip are: $w_{0}=3030 \mu \mathrm{m}, w_{1}=433 \mu \mathrm{m}, l=800 \mu \mathrm{m}, d=179 \mu \mathrm{m}$.

A complete theoretical analysis of the flow characteristics as a function of pressure drop variations along the flow direction in such a geometry has been provided in [30]. The approach consists to measure the pressure drop along a progressive contraction flow and separate the contribution due to shear stresses from that due to extensional stresses. This geometry finally allows us to obtain a good estimation of the apparent planar extensional viscosity, but in the following we simply refer to it as the extensional viscosity.

In order to appreciate the extensional behavior of our fluids we focus on the (apparent) Trouton number $(T r)$, i.e. the ratio of the apparent extensional viscosity to the apparent shear viscosity. For a Newtonian fluid in planar extension $T r$ is equal to 4 [31]. In Figure 4 the value of $T r$ is shown as a function of the strain rate for some of our fluids. Since we could make measurements only in a limited range of strain rates the present information is partial but we can already see that for our materials $T r$ increases with polymer concentration and with strain rate up to values much larger than 4 . 


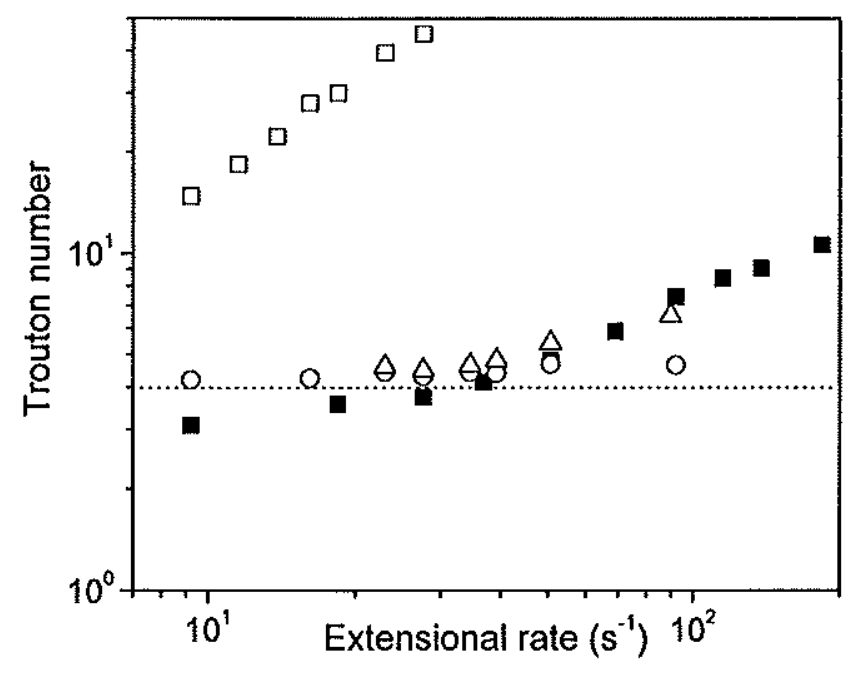

Figure 4: Trouton number as a function of apparent extensional rate for different materials: PAM6000 (filled squares), PEGPEO-250 (circles), PEGPEO-500 (triangles), PEGPEO-2000 (squares).

\subsection{Channelized flow experiments}

Our experimental set up consists in a cell, i.e. a rectangular cross section channel (the main channel) made of glass (thickness: $b=2 \mathrm{~mm}$; width: $D=2 \mathrm{~cm}$ ) (see Figure 5) through which the Newtonian oil ( $\eta=0.1$ Pa.s) is pushed by different types of displacing fluids.

Before the displacement experiment begins, the channel is partly filled with the displacing fluid (bottom), then the Newtonian oil (displaced fluid) is introduced by the top of the cell and comes into contact with the first fluid so that we do not have remaining air in the cell. 


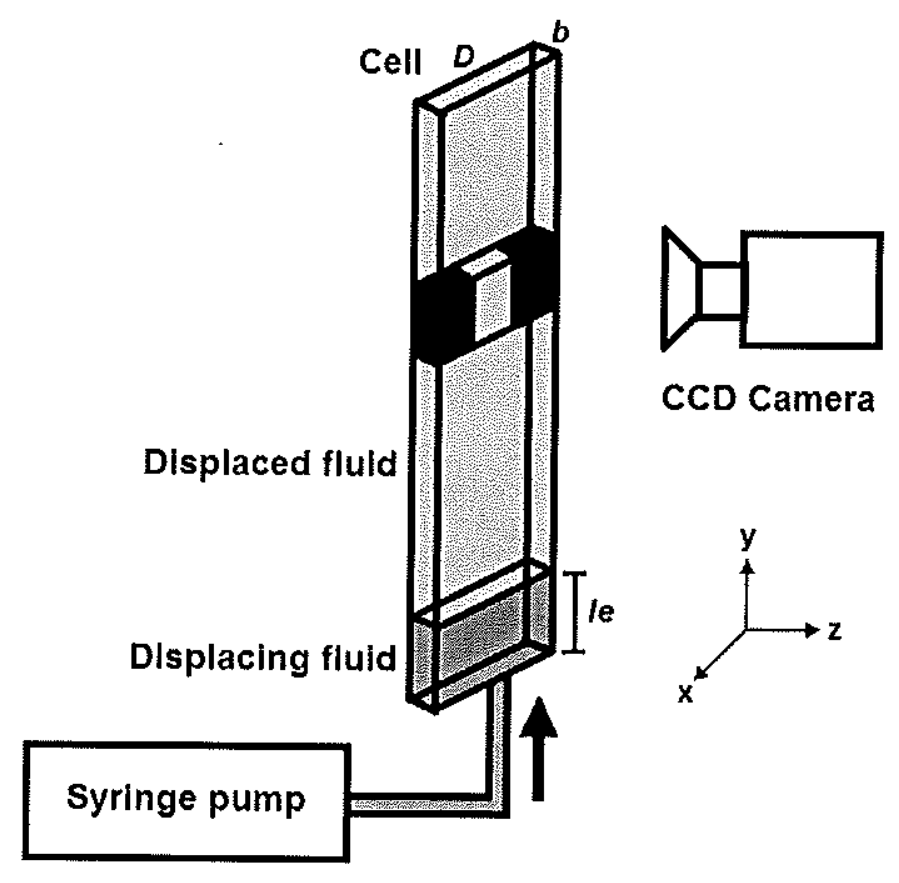

Figure 5: Scheme of the experimental set-up.

After the set up of the two fluids as described above a displacement experiment begins (initial time) with the injection, at a constant flow rate $Q$, of the displacing fluid from the lower inlet using a multiple syringe pump system (KDS220, KD Scientific) which allows us to induce a maximum flow rate of 12 $\mathrm{ml} / \mathrm{s}$. This device pushes the oil vertically through the channel (see Figure 5) and induces along the channel an average velocity $V \approx Q / b D$. In the following we will use this velocity as a reference characteristics of the flow. In our tests a Reynolds number associated with the mean flow characteristics could be computed from the following expression: $\operatorname{Re}=\rho V b / \eta$, in which $\rho$ is the fluid density. In this expression, for a non-Newtonian fluid we used the value of the apparent viscosity of this fluid for the apparent shear rate of the flow $(\overline{\dot{\gamma}})$ defined in Section 4.1.

The flow characteristics are observed from the initial time via the evolution of the apparent interface from above the largest channel side (plane $(x, y)$ ). The images are acquired with a CCD Camera monochrome CV-M10 $50 \mathrm{~Hz}$ equipped with a macro-objective. The illumination was obtained thanks to a fibre optic illuminator behind the cell with a "white screen". The images were processed with MATLAB in order to obtain the interface profiles. Note that this is only an apparent interface that we can observe with this technique. The effective interface is likely curved along the $\mathrm{z}$-axis, so that the observed shape might correspond to the position of the front line of the interface along the $y$-axis, in other words the intersection of the interface and the plane $\mathrm{z}=0$. In the following we will refer to this line as the 
"interface". The reproducibility of the whole flow process and interface image processing was found to be excellent: two interface profiles obtained from two different tests under the same experimental conditions cannot be distinguished from each other:

As described in [25] after a relatively short distance (say, about $5 \mathrm{~cm}$ ) the profile of the interface between the two fluids appears to be stationary. This means that the fluid approximately has a uniform velocity along the channel width when it approaches the contraction situated much farther (see below). Moreover the shape of this stationary profile depends on the rheological behavior of the displacing fluid. It was shown that for a Newtonian fluid the profile becomes sharper for increasing velocity while for a fluid exhibiting significant normal stress it remains similar to that for a Newtonian fluid at low velocity (say, around $1 \mathrm{~cm} . \mathrm{s}^{-1}$ ). An example is shown in Figure 6. It seems that these relatively small differences in the profile shape cannot explain the much more significant differences observed in the interface deformation during the flow through the contraction (see below).

a)

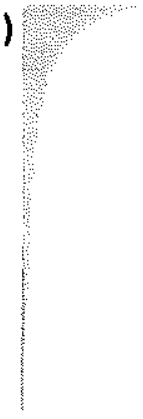

b)

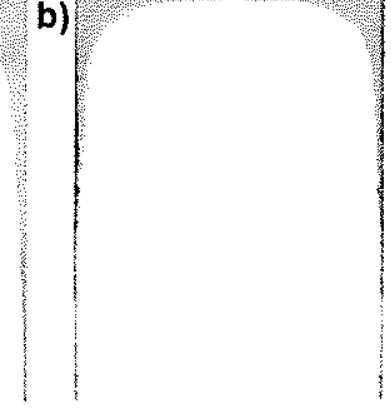

Figure 6: Stationary interface profile at $5 \mathrm{~cm} \cdot \mathrm{s}^{-1}$ for a) Canadou and b) PAM6000.

A restriction of the channel is positioned at a distance $l e=20 \mathrm{~cm}$ from the initial position of the interface, i.e. at a distance equal to 10 times the width of the largest channel. The restriction has a rectangular shape: it simply reduces the channel width to $D^{\prime}=0.67 \mathrm{~cm}$ over a distance of $4 \mathrm{~cm}$. The central channel will be called the small channel in the following.

\section{Results}

For all experimental conditions we look at the interface evolution in time during the fluid displacement from a series of successive pictures. The first picture is taken when the interface front just reaches the bottom of the window of observation. The next pictures are taken at successive times separated by a 
period $\Delta t=\Delta l / V$, where $\Delta l$ is a constant distance equal to $4 \mathrm{~mm}$. Under such conditions we can compare the interface evolution at different velocities and after different times of flow associated with the same global displacement of the displacing fluid.

\subsection{Newtonian displacing fluid}

The first interface profile (see Figure 7a), situated at a distance of about $1 \mathrm{~cm}$ from the channel entrance, is the stationary front profile observed upstream only slightly deformed by the approach of the contraction. Then, as we approach the restriction at a close distance the fluid forms a central finger which penetrates through the channel (see Figures $7 b, c$ ). The finger width is thinner for higher velocities (see Figure 7).
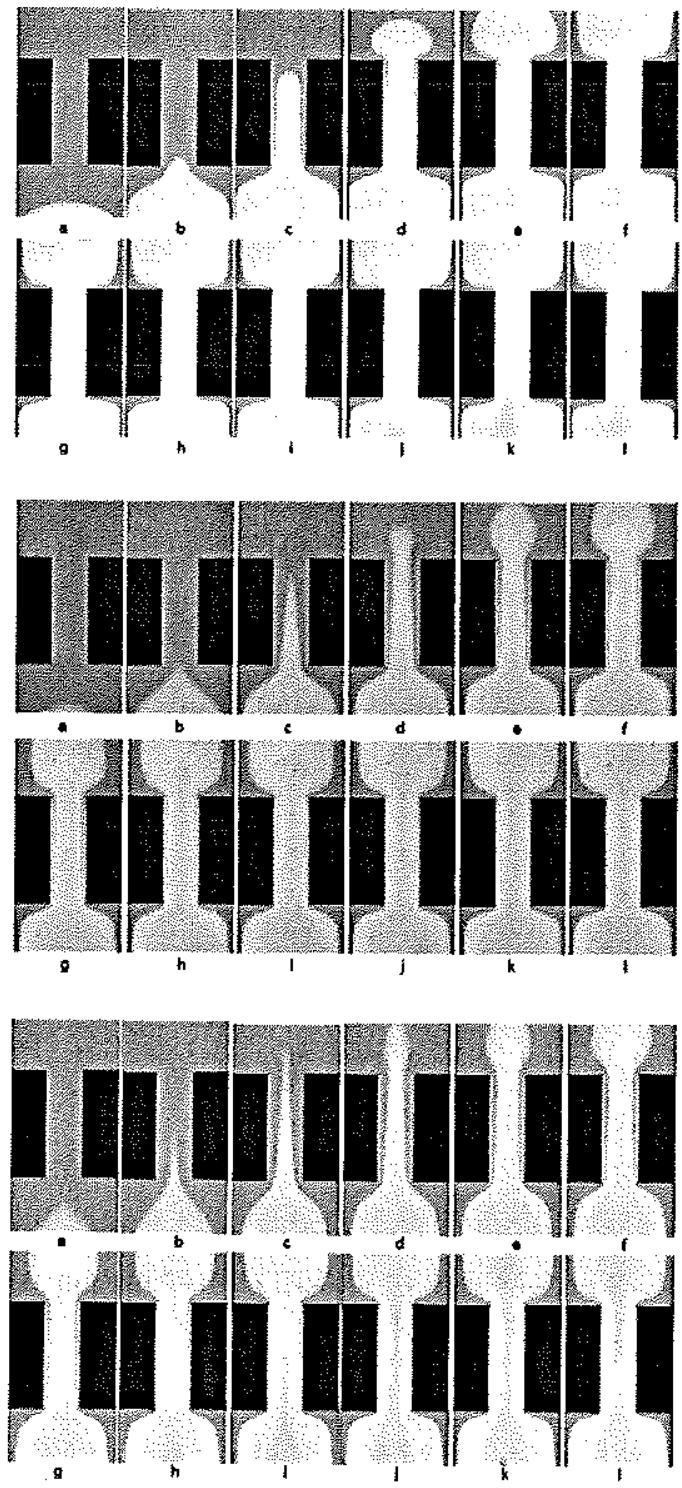
Figure 7: Successive views (from (a) to (I)) of the flow through the restriction with a time period corresponding to an average displacement of $4 \mathrm{~mm}$ for a Newtonian displacing fluid at different velocities: (top) $1 \mathrm{~cm} \cdot \mathrm{s}^{-1} \quad(\operatorname{Re}=0.17), \quad$ (center) $5 \mathrm{~cm} \cdot \mathrm{s}^{-1}(\operatorname{Re}=0.83)$, (bottom) $10 \mathrm{~cm} \cdot \mathrm{s}^{-1}$ $(\operatorname{Re}=1.7)$.

Then the displacing front reaches the exit of the channel and starts to expand, forming first a mushroomlike shape which is narrower at higher velocities (see Figure 7d). As time goes on the size of this mushroom increases and finally the downstream width of the displacing fluid region (at the top of the window) reaches the sides of the large channel (see Figures $7 \mathrm{e}, \mathrm{f}, \mathrm{g}$ ). During that time the interface profile eventually reaches an apparently stationary shape (see Figures $7 \mathrm{~h}, \mathrm{i}, \mathrm{j}, \mathrm{k}, \mathrm{l}$ ) for which the finger has now fully invaded the small channel. The global displacement needed to reach this stationary regime slowly increases from about 3 to $4 \mathrm{~cm}$ when the velocity increases from 1 to $10 \mathrm{~cm} . \mathrm{s}^{-1}$. Looking at the interface shape for an additional global displacement of the same order we did not observe a further significant change of the interface.

\subsection{Viscoelastic displacing fluid}

With a viscoelastic displacing fluid the overall flow characteristics are similar: stretching of the interface, penetration into the small channel, widening at the exit, then progressive establishment of the steady state (see Figure 8). This stationary regime is obtained approximately under the same conditions as for a Newtonian fluid, i.e. after a global displacement slightly increasing with the velocity from about 3 to 4 $\mathrm{cm}$. Let us recall that this is only an apparent stationary regime within the maximum duration of our observation, imposed by the maximum volume of fluid which could be injected (typically associated with a global displacement of about $30 \mathrm{~cm}$ ).

\section{Impact of velocity}

At low velocity, i.e. here $1 \mathrm{~cm} . \mathrm{s}^{-1}$, the interface deformations are similar to those observed for the Newtonian fluid (see Figures 7 and 8 top). The finger size, the mushroom shape and the stationary shape of the interface are very similar for the two fluid types, the only difference lying in the width of the transient finger in the small channel which is larger for the viscoelastic fluid.

At first sight at a higher velocity (i.e. $5 \mathrm{~cm} . \mathrm{s}^{-1}$ ) (see Figure 8 center) we have approximately the same effects, but the stationary interface profile starts to slightly differ from that in the Newtonian case in the entrance region (see Figure 7). Moreover the width of the finger in the small channel and the mushroom 
shape are approximately the same as for a smaller velocity, which contrasts with our observations in the Newtonian case (strong evolution of the initial finger width with velocity). This suggests that viscoelastic effects could tend to maintain a wide lateral extent of the displacing fluid.

The above effects become clearer at a higher velocity (i.e. $10 \mathrm{~cm} \cdot \mathrm{s}^{-1}$ ). Now the displacing fluid in the small channel roughly occupies the same width as for smaller velocities but its aspect is that of a series of parallel fingers (see Figure $9 \mathrm{~d}$ bottom) which suggests that some kind of instability occurs during this phase. Note that although this effect looks like a diffusive penetration of the displacing fluid through the other fluid this cannot be the case because the two fluids are immiscible; the progressive color variation towards the finger tips is rather due to a gradient (decrease) of their thickness (along $z$ ). Moreover in the stationary regime the interface extends more widely for a larger velocity: it progressively come into contact with a wider area along the exit sides of the small channel (see Figure 81 top, center, bottom). These effects mean that when one increases the velocity a viscoelastic fluid tends to spread more widely than a Newtonian fluid through and after the restriction. On the contrary, at higher velocity the displacing fluid tends to occupy a thinner region before the small channel entrance (see Figure 81, top, center, bottom).

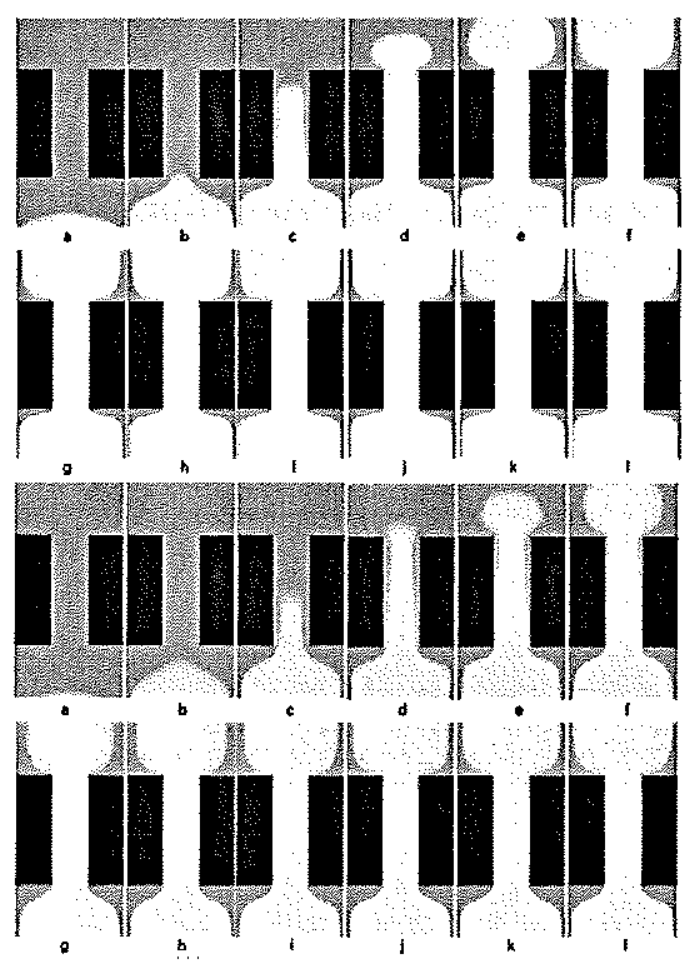




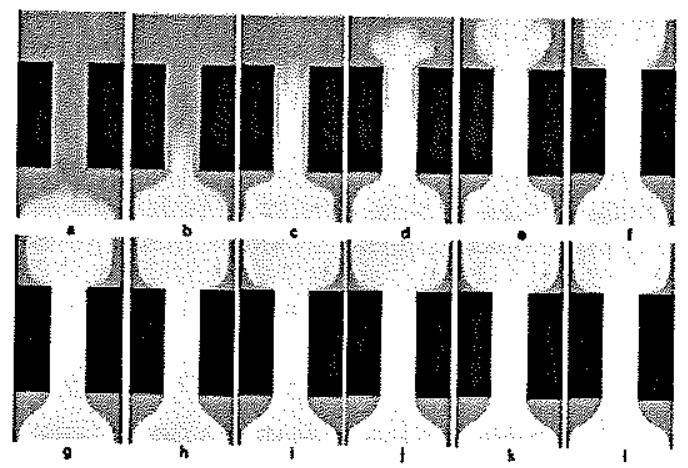

Figure 8: Successive views (from (a) to (l)) of the flow through the restriction with a time period corresponding to an average displacement of $4 \mathrm{~mm}$ for PAM6000 displacing fluid at different velocities: (top) $1 \mathrm{~cm} \cdot \mathrm{s}^{-1}(\mathrm{Re}=0.05 ; \mathrm{We}=1.2)$, (center) $5 \mathrm{~cm} \cdot \mathrm{s}^{-1}(\mathrm{Re}=0.36 ; \mathrm{We}=6)$, (bottom) $10 \mathrm{~cm} \cdot \mathrm{s}^{-1}(\operatorname{Re}=0.87 ; \mathrm{We}=12)$.

\section{Impact of polymer concentration}

The trends which appear for a given polymer solution when increasing the velocity also appear at a given velocity when increasing the polymer concentration. Indeed for a PEGPEO at $500 \mathrm{ppm}$ all the flow characteristics at the different stages are similar to the Newtonian case for the same velocities. Then, when the polymer concentration of the PEGPEO solution is increased we observe the different trends previously observed for PAM6000 when increasing the velocity. Here we illustrate these trends only through the pictures at a velocity $10 \mathrm{~cm} \cdot \mathrm{s}^{-1}$ for PEGPEO at different concentrations (see Figure 9).

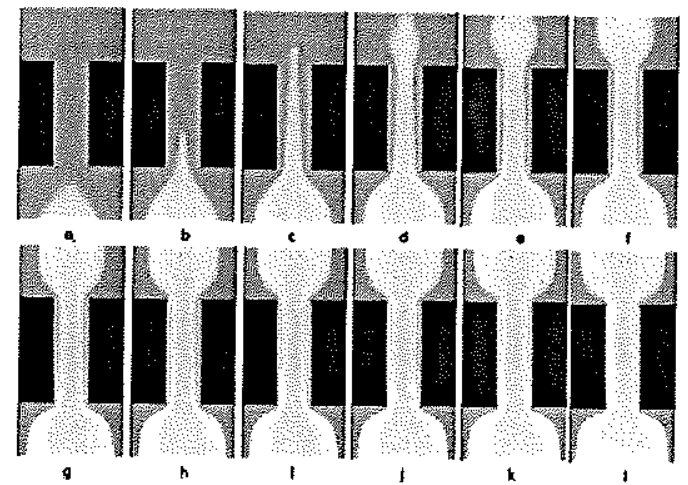



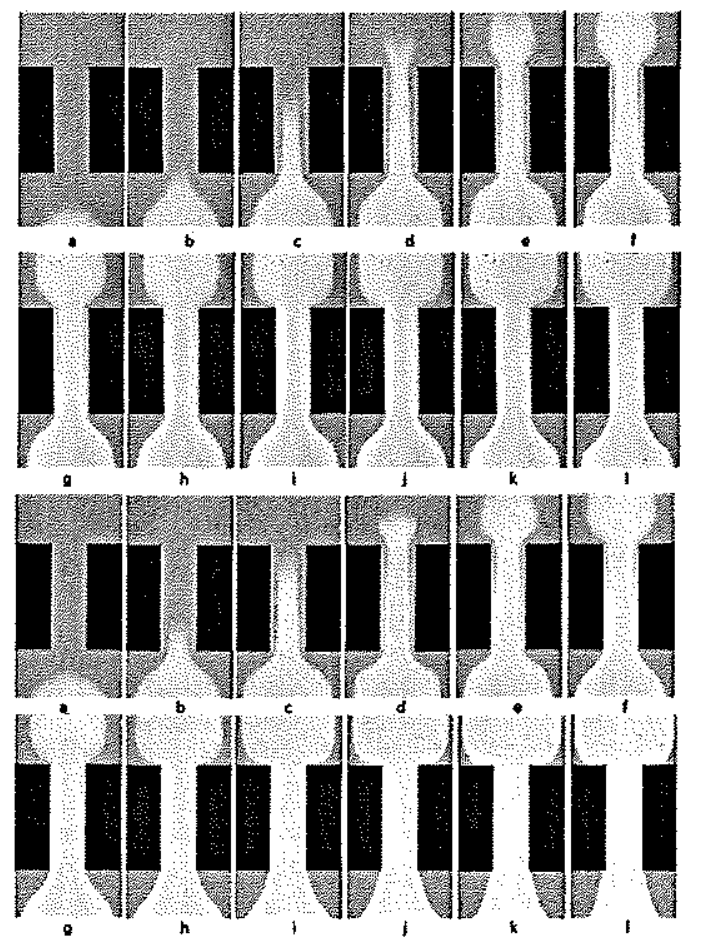

Figure 9: Successive views (from (a) to (l)) of the flow through the restriction with a time period corresponding to an average displacement of $4 \mathrm{~mm}$ at an average velocity $10 \mathrm{~cm} . \mathrm{s}^{-1}$ for PEGPEO displacing fluid at different concentrations: (top) $500 \mathrm{ppm}(\mathrm{Re}=1 ; \mathrm{We}=6)$, (center) $1000 \mathrm{ppm}$ $(\mathrm{Re}=0.87 ; \mathrm{We}=17.4)$, (bottom) $2000 \mathrm{ppm}(\mathrm{Re}=0.74 ; \mathrm{We}=36)$.

We could also observe an additional effect for PEGPEO-2000 at velocities larger than $10 \mathrm{~cm} / \mathrm{s}$ and PEGPEO-3000 for velocities larger than $5 \mathrm{~cm} / \mathrm{s}$. In that case the flow starts by developing as described above for high velocity or high polymer concentration (see Figure 10). It seems to reach the steady state shape obtained in such a case, i.e. with a thinning of the flow width before the entrance and a swelling at the exit. Then the region occupied by the viscoelastic fluid slightly widens before the entrance and some instability develops, leading to the formation of two small vortices close to the entrance corners (see Figure 10t to $\mathrm{x}$ ).

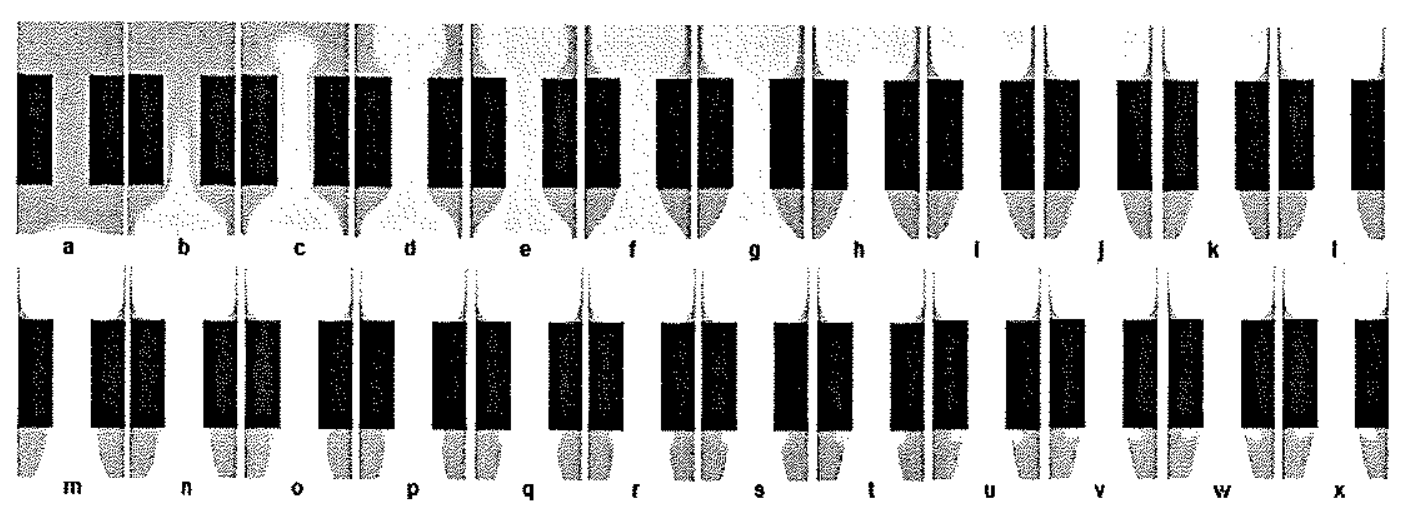


Figure 10: Successive views (from (a) to (x)) of the flow through the restriction with a time period corresponding to an average displacement of $4 \mathrm{~mm}$ at an average velocity $10 \mathrm{~cm} \cdot \mathrm{s}^{-1}$ for PEGPEO3000 displacing fluid $(\operatorname{Re}=0.7 ; \mathrm{We}=70)$.

\subsection{Steady state flow characteristics}

We can now specifically focus on the final pictures obtained in the stationary regime for the different fluid types at different velocities (see Figure 11). First we remark that whatever the displacing fluid type it does not invade all the volume initially occupied by the Newtonian oil (see Figure 11a). There remains a significant volume of static oil in the corners before the entrance and after the exit of the restriction. On the contrary the small channel is apparently fully invaded by the displacing fluid.

For the Newtonian displacing fluid the shape of the interface is symmetrical with regards to a central horizontal line (see Figure 1la). The same result is obtained when viscoelastic effects are a priori negligible (sufficiently low velocity and low polymer concentration) (see Figure 11 a,c). On the contrary at sufficiently high velocity and polymer concentration a dissymmetry in the interface profile appears in the steady state profile (see Figure $11 \mathrm{~b}, \mathrm{~d}, \mathrm{e}$ ). More precisely, before the entrance the profile tends to progressively adjust to the channel width, which leads to a large remaining volume of oil in this region. On the contrary at the exit the displacing fluid abruptly tends to occupy a widest volume and comes into contact with the exit sides of the small channel. These trends and the resulting dissymmetry in the interface profile are more marked for increasing velocity and increasing polymer concentration (see Figure $11 \mathrm{c}, \mathrm{d}, \mathrm{e})$.
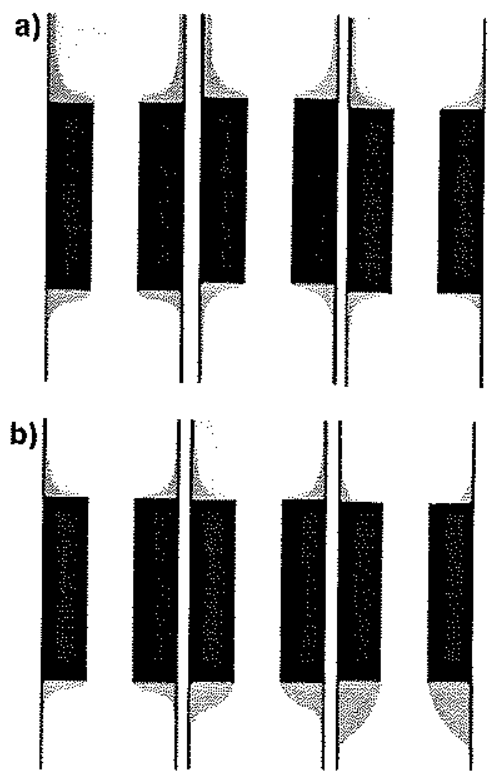

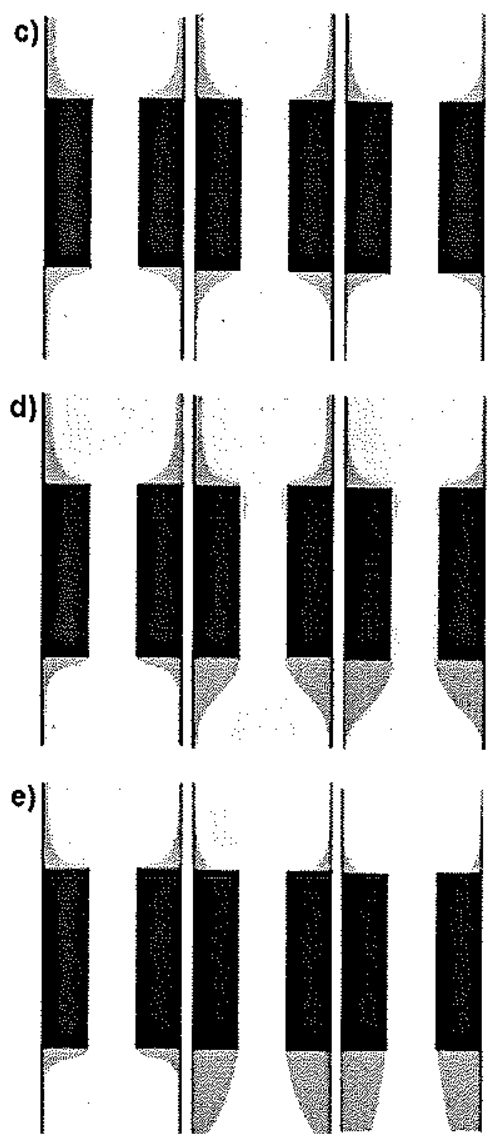

Figure 11: Steady state views of the flow through the restriction at different velocities ((from left to right) $1 \mathrm{~cm} . \mathrm{s}^{-1}, 5 \mathrm{~cm} . \mathrm{s}^{-1}$, and $10 \mathrm{~cm} . \mathrm{s}^{-1}$ ) for different displacing fluids: (from top to bottom) Newtonian fluid, PAM6000, and PEGPEO at 500, 1000 and $2000 \mathrm{ppm}$.

\section{Discussion}

\subsection{Flow characteristics analysis}

Let us consider the flow at a sufficient distance from the restriction. In that case it was observed that the interface approximately keeps a constant shape at least over a channel length of about $10 \mathrm{~cm}$ [25]. As a consequence we have essentially a simple shear in this region: the velocity of the fluid elements is mainly directed along the main channel axis. In order to estimate the range of shear rates involved in this flow we can compute the apparent shear rate by simply dividing the average velocity by the distance between the plates: $\overline{\dot{\gamma}}=V / b$. Obviously we expect that the effective shear rate in the fluids ranges from lower values than $\bar{\gamma}$ far from the walls to larger values along the walls.

For the steady state flow of a single Newtonian fluid between two parallel plates we can solve the momentum equation and find the velocity profile from which we deduce the following relationship between this apparent shear rate and the (maximum) shear rate at the wall: 


$$
\dot{\gamma}_{m}=6 \overline{\dot{\gamma}}
$$

If the flow in the small channel was established (with a constant interface profile) and could be considered as the flow between two wide parallel plates we would have corresponding values three times larger for a Newtonian fluid since due to the mass conservation the flow rate is three times larger in this channel. For the steady state uniform flow of a single viscoelastic fluid exhibiting a constant viscosity in steady-state, whatever the normal stress the velocity profile is the same as for a simple Newtonian fluid. Indeed the normal stress component is absent of the momentum equation for the shear stress, which has thus the usual form in the Newtonian case.

As soon as we are dealing with a two-phase flow or a more complex geometry the above results obtained with a single fluid are no longer strictly valid. In particular, for our more complex flows the effective maximum shear rate likely differs from the above expected values for a Newtonian uniform flow around the entrance or exit of the restriction. We can also have some wall slip resulting from a dewetting along the line of contact between the two fluids and the solid surface. Moreover, since the channel width is finite there is some shear induced by the solid side walls which gives rise to the deformation of the interface that we observe. If we consider that the shear along the channel width is approximately that due to the uniform flow between two infinite plates at a distance of $D$ from each other we deduce that the apparent shear rate due to the presence of channel sides in the main channel is equal to $(b / D) \dot{\gamma}=\dot{\gamma} / 10$.

Due to these various complex aspects it is not possible to find in a straightforward way a single relevant characteristic shear rate value associated with the flow with a specific displacing fluid at a given velocity. However it is simpler to deal with a single value than with a range of shear rates. As a consequence in the following we will somewhat arbitrarily associate to each velocity a typical shear rate by computing the wall shear rate $\left(\dot{\gamma}_{m}\right)$ in the upstream uniform Newtonian flow: for a velocity of 1,5 and $10 \mathrm{~cm} . \mathrm{s}^{-1}$ we thus obtain respectively: 30,150 and $300 \mathrm{~s}^{-1}$.

Note that around the entrance in the small channel there is also a significant extensional flow component since the channel width decreases by a factor 3 . The flow is complex as it involves both extensional flow and shear flow in three dimensions. We can estimate the apparent strain rate resulting from the velocity change by assuming that a length of channel $l$ and moving at an average velocity $V$ is elongated by a factor $D / D^{\prime}$ when flowing through the small channel so that we obtain:

$\bar{\varepsilon}=\frac{V}{l}\left(\frac{D}{D^{\prime}}-1\right)$ 


\subsection{Transient regime}

Let us first consider the case of a Newtonian displacing fluid. Our results concerning the impact of the velocity on the width of the finger in the small channel, which decreases with velocity increase, is reminiscent of the typical trend observed for the Saffman-Taylor instability. However this instability cannot take place here because the displacing fluid viscosity is (slightly) larger than the displaced fluid viscosity. Indeed in this instability a digitation develops because this process minimizes the viscous dissipation. As a consequence the apparent fingering process observed at sufficiently large velocity is likely mainly induced by the presence of the restriction downstream and the complex two-phase flow which is induced, in a way that we are unable to explain further. We can observe a strong difference with a viscoelastic fluid at sufficient high polymer concentration or velocity: now the finger width remains close to the channel width. Once again we are unable to explain in depth this effect, it can only be suggested that due to normal stress effects the displacing fluid tend to expand transversally through the displaced fluid.

\subsection{Stationary regime}

In the Newtonian case the stationary shape of the interface is symmetrical and independent of the velocity (see Figure 11). Such a situation is expected for the monophasic (Stokes) flow of a Newtonian fluid at sufficiently low Reynolds number and with negligible interfacial effects because when only viscous effects play the major role, as the stress is increased everywhere by a single factor the velocity is increased everywhere by the same factor. In our case the fact that we obtain qualitatively the same result as for a monophasic flow, namely an independence of the interface profile on velocity, suggests that there is no impact of surface tension effects with regards to viscous effects. This conclusion is also supported by the fact that we obtain similar evolutions of the interface for viscoelastic and Newtonian fluids at low velocities whereas the interfacial tension is not the same for these different fluids. However it is worth emphasizing that due to the presence of two immiscible fluids in contact (i.e. the existence of an interface) the aspect of the interface shape in the stationary regime cannot be directly compared with the contour of the streamlines observed for a single Newtonian fluid, such as those in a square-square contraction (see [32]). Indced there tends to remain a small layer of displaced fluid between the displacing fluid and the lateral walls. It is not clear whether this layer will tend to disappear after much larger times of flow. Nevertheless, if we leave apart this aspect the overall interface shape in our case is qualitatively similar to the contour of streamlines for a single Newtonian fluid at low Reynolds number.

An interesting result in our data is the existence of some non-displaced material in the corners before and after the small channel. Actually it is well known that for a monophasic flow of a Newtonian fluid 
through a complex geometry there can exist regions of reversed vorticity in which there is no net flux along the main flow direction [33]. This suggests that for a two-phase flow, in the absence of specific interfacial effects, the interface between the two simple fluids could approximately take the position of the surface of separation between the flowing and reversed vorticity regions for a monophasic flow through the same geometry.

On the contrary, with viscoelastic fluids the interface profile starts to be dissymmetrical for sufficiently large velocity and sufficiently large polymer concentration. This obviously suggests that this effect is related to the importance of elastic effects. It is natural to analyze such data by computing the Weissenberg number $(W e$ ) which compares viscous effects to elastic effects. Here we compute the Weissenberg number from the characteristic shear rate estimated above and the relaxation time of the fluid: $W e=\lambda \dot{\gamma}_{m}$. The values of this number for the different flows and materials are represented in Figure 12. In this figure the flows for which we clearly see a reduction of the width of the displacing fluid before the entrance with regards to the width for a Newtonian fluid, are situated above a slightly inclined (dashed) line. This means that from our data this situation corresponds to a critical value of the Weissenberg number which could slightly vary with the velocity but is around 5.5 in our range of tests. Also note that we drew a dashed line to separate flows without vortex from those with vortex. Once again the transition seems to occur around a critical We number value (say 40).

Actually it seems natural to associate this reduction of the displacing fluid extent before the entrance to extensional effects. Indeed we can suspect that if the viscous dissipation due to the extensional effects before the entrance becomes sufficiently large the flow width will adjust to minimize them. More precisely if this viscous dissipation increases rapidly with the strain rate it would become preferable for the fluid to decrease the strain rate by extending the region of transition (i.e. increasing $I$ ). In our case, when the length of fluid $l$ is equal to $4 \mathrm{~mm}$ the extensional rate is equal to 5,25 and $50 \mathrm{~s}^{-1}$ for the three velocity levels. If we look at the Trouton number values we see that extensional effects are negligible for PEGPEO at $500 \mathrm{ppm}$ in this strain rate range. For PEGPEO-2000 and PAM6000 we have a Trouton number much larger than expected for a Newtonian one but this is true for all the strain rate levels tested here so that we would expect extensional effects also for the smallest velocity, in contrast with our data. This means that our interpretation is only partially validated by the data. 


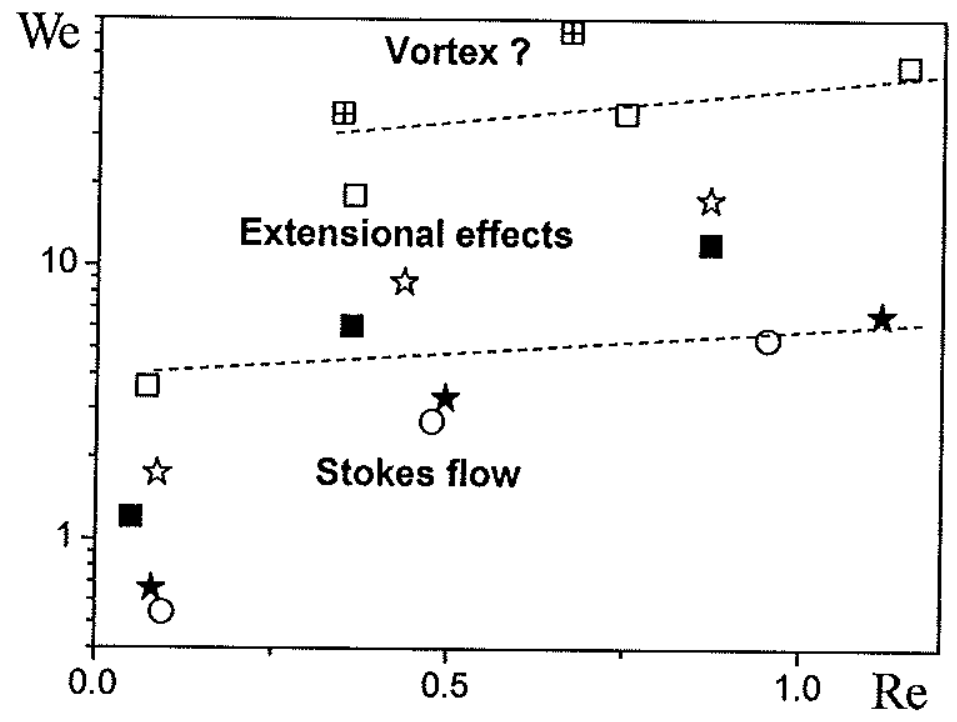

Figure 12: Weissenberg number as a function of the mean flow rate for flows of PAM6000 (filled squares), PEGPEO at 500 (circles), 1000 (stars), $2000 \mathrm{ppm}$ (open squares), and $3000 \mathrm{ppm}$ (crossed squares). The lower dashed line separates flows apparently affected by elastic effects (here flow width reduction before the entrance) and those which are unaffected ("Stokes flow": steady state flow characteristics similar to the Newtonian case). The upper dashed line separates flow without vortex from those with vortex.

Let us now consider the trend observed at the exit of the small channel. We would expect the same effect observed at the entrance but in the opposite direction, i.e. a gradual expansion of the displacing fluid width in order to minimize dissipation caused by extensional effects. But the opposite trend is observed: a widening, even more important as velocity or concentration increases. Obviously such a result cannot be explained by extensional effects.

We suggest that normal stress effects are at the origin of the phenomenon. In order to check this interpretation we first need to characterize it more precisely: here we will consider that the effect unambiguously takes place when the interface between the two fluids at the exit of the small channel is apparently (from our pictures) in contact with the solid sides over a distance larger than $2 \mathrm{~mm}$. On the contrary, for a Newtonian fluid or when viscoelastic effects are negligible the interface is clearly not in contact with the solid sides (see Figure 11).

Obviously normal stresses can play a significant role only if they are sufficiently large with regards to tangential stresses. As a consequence the relevant parameter for studying their impact is the ratio of the normal stress to the shear stress, i.e. $\chi=N_{1} / \tau$. In Figure 13 we plotted the value of this parameter for the different fluids as a function of the characteristic shear rate in our flows. A single line can be drawn to 
separate the flows affected by elastic effects according to the above criterion from those which are unaffected. It appears that this line is situated around a value of the order of 1 . This suggests that the transition towards the swelling effect occurs when the normal stress become larger than the tangential stress. The problem is that we do not observe a single transition location for the two different fluids, which suggests that there might some additional effects playing a role in the process.

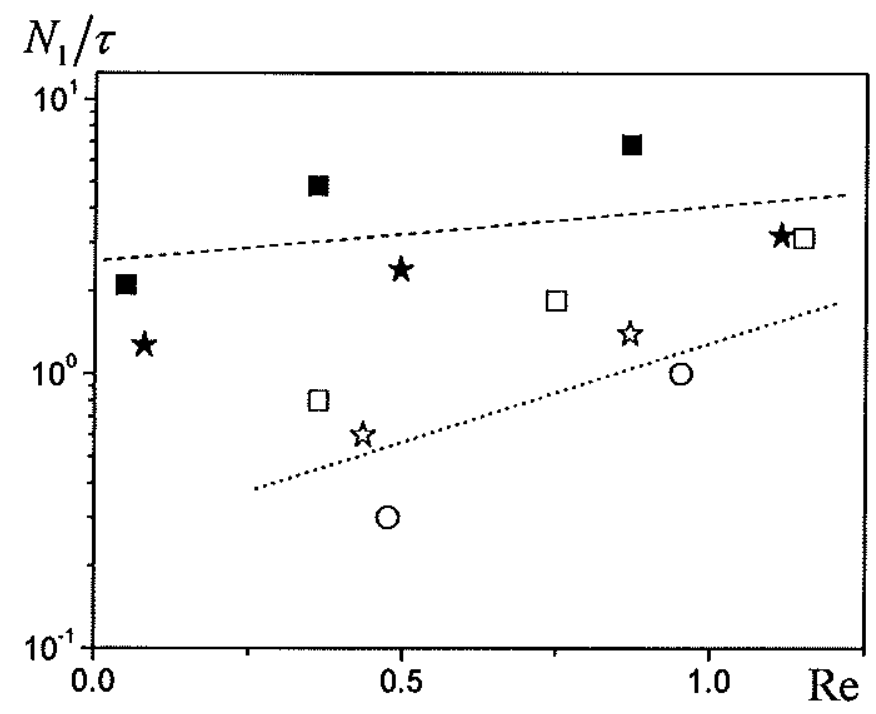

Figure 13: Normal to shear stress ratio as a function of the Reynolds number for flows of PAM4000 (filled stars), PAM6000 (filled squares), PEGPEO at 500 (circles), 1000 (stars) and $2000 \mathrm{ppm}$ (open squares). Flows apparently affected by elastic effects (here swelling at the exit of the small channel) and those unaffected are separated by: a dashed line (PAM) or a dotted line (PEGPEO).

\section{Conclusion}

The stationary shape of the interface between a viscoelastic fluid flowing through a simple liquid and through a rectangular contraction appears to have characteristics reminiscent of the velocity field of a single viscoelastic fluid through a contraction. For low Weissenberg number the shape of the interface is essentially similar to that between two simple liquids, the shape is symmetrical (entrance and exit shape) and there are dead (likely recirculating) regions in the corners. For sufficiently large Weissenberg number the shape of the interface is dissymmetrical: it is narrower before the entrance and wider just after the exit; the dead regions in the entrance corners increase in size with the Weissenberg number while those in the exit corners decrease in size. This suggests that the entrance effects in this two-phase flow situation are due to extensional effects and the exit effects are due to normal stress effects. It is also worth mentioning that some instability occurs at even larger Weissenberg number, which leads to the 
development of vortices just on the sides of the entrance in the contraction. This effect might be specific of such a two-phase flow with non-Newtonian fluid but still need to be fully characterized.

A question of interest is how these results can be extrapolated to a porous medium, and in particular if it means that a viscoelastic fluid tends to better remove the liquid contained in the medium. The answer is still not simple. If we consider that a porous medium structure is made of a series of contractions our results suggest at first sight that in the stationary regime there will remain approximately the same amount of pre-existing liquid for a Newtonian or viscoelastic displacing fluid since in the latter case the smaller amount of remaining liquid after the exit is roughly balanced by a larger amount before the entrance.

However in the transient regime, i.e. during the initial penetration of the displacing fluid through the simple one, the shape of the interface is significantly wider for a viscoelastic fluid at sufficiently high Weissenberg numbers (see Figures 9, 10 and 11). This means that the amount of simple liquid displaced during this stage is clearly larger than for a Newtonian displacing fluid. Our results thus suggest that using a viscoelastic displacing fluid at high Weissenberg numbers will lead to the same remaining quantity of liquid as for a Newtonian displacing fluid but this situation will be reached faster.

\section{References}

[1] R. Seright, J. Seheult, T. Talashek, Injectivity Characteristics of EOR Polymers, SPE Reservoir evaluation \& Engineering, 12, 783-792 (2009)

[2] P.G. Saffman, G. Taylor, The Penetration of a Fluid into a Porous Medium or Hele-Shaw Cell Containing a More Viscous Liquid, Proc. Royal Soc. Lond. Ser. A. Math Phys. Sci. 245 (1958) 312 -329.

[3] L. Zhang, X. Yue, F. Guo, Micro-mechanisms of residual oil mobilization by viscoelastic fluids, Pet. Sci. 5 (2008) 56-61.

[4] W. Demin, J. Youlin, W. Yan, X.H. Gong, W. Gang, Viscous-elastic polymer fluids rheology and its effect upon production equipment, SPE Production \& Facilities, 19, 209-216 (2004)

[5] D. Wang, G. Wang, W. Wu, H. Xia, H. Yin, The Influence of Viscoelasticity on Displacement Efficiency-From Micro- to Macroscale, SPE Annual Technical Conference and Exhibition, 11-14 November 2007, Anaheim, California, U.S.A.

[6] C. Zhang, M. Oostrom, T.W. Wietsma, J.W. Grate, M.G. Warner, Influence of Viscous and Capillary Forces on Immiscible Fluid Displacement: Pore-Scale Experimental Study in a Water-Wet Micromodel Demonstrating Viscous and Capillary Fingering, Energy Fuels. 25 (2011) 3493-3505.

[7] R. Lenormand, C. Zarcone, A. Sarr, Mechanisms of the displacement of one fluid by another in a network of capillary ducts, J. Fluid Mech. 135 (1983) 337-353.

[8] R, Lenormand, E. Touboul, C. Zarcone, Numerical models and experiments on immiscible displacements in porous media, J. Fluid Mech. 189 (1988) 165-187. [9] S.D.R. Wilson, The Taylor-Saffman problem for a non-Newtonian liquid, J. Fluid Mech. 220 (1990) $413-$
425 .

[10] A. Lindner, D. Bonn, E.C. Poiré, M.B. Amar, J. Meunier, Viscous fingering in non-Newtonian fluids, J. Fluid Mech. 469 (2002) 237-256.

[11] R. Srinivasan, The hole pressure problem: On the Higashitani-Pritchard theory for transverse and axial slots, Rheol. Acta. 26 (1987) 107-118. 
[12] S.H. Spiegelberg, G.H. McKinley, Stress relaxation and elastic decohesion of viscoelastic polymer solutions in extensional flow, J. Non-Newton. Fluid Mech. 67 (1996) 49-76.

[13] L. Choplin, P.J. Carreau, End effects for highly elastic-constant viscosity fluids, Rheol. Acta. 25 (1986) 95101.

[14] G.H. McKinley, W.P. Raiford, R.A. Brown, R.C. Armstrong, Nonlinear dynamics of viscoelastic flow in axisymmetric abrupt contractions, J. Fluid Mech. 223 (1991) 411 456.

[15] L.E. Rodd, J.J. Cooper-White, D.V. Boger, G.H. McKinley, Role of the elasticity number in the entry flow of dilute polymer solutions in micro-fabricated contraction geometries, J. Non-Newton. Fluid Mech. 143 (2007) $170-191$.

[16] L.E. Rodd, D. Lee, K.H. Ahn, J.J. Cooper-White, The importance of downstream events in microfluidic viscoelastic entry flows: Consequences of increasing the constriction length, J. Non-Newt. Fluid Mech. 165 (2010) 1189-1203.

[17] L. Campo-Deano, F.J. Galindo-Rosales, F.T. Pinho, M.A. Alves, M.S.N. Oliveira, J. Non-Newt. Fluid Mech., $166,1286-1296(2011)$

[18] A.S. Lubansky, D.V. Boger, C. Servais, A.S. Burbidge, J.J. Cooper-White, An approximate solution to flow through a contraction for high Trouton ratio fluids, J. Non-Newt. Fluid Mech. 144 (2007) 87-97.

[19] V. Ganvir, A. Lele, R. Thaokar, B.P. Gautham, Simulation of viscoelastic flows of polymer solutions in abrupt contractions using an arbitrary Lagrangian Eulerian (ALE) based finite element method, J. NonNewton. Fluid Mech. 143 (2007) 157-169.

[20] S. Yoon, Y. Kwon, Finite element analysis of viscoelastic flows in a domain with geometric singularities, Korea-Aust. Rheol, J. 17 (2005) 99-110.

[21] G.N. Rocha, R.J. Poole, P.J. Oliveira, Bifurcation phenomena in viscoelastic flows through a symmetric 1:4 expansions, J. Non-Newt. Fluid Mech., 141, 1-17 (2007)

[22] P.C. Sousa, P.M. Coelho, M.S.N. Oliveira, M.A. Alves, Laminar flow in three-dimensional square-square expansions, J. Non-Newtonian Fluid Mech., 166 (2011) 1033-1048.

[23] H.A. Barnes, J.F. Hutton, K. Walters, An Introduction to Rheology, Elsevier, 1989.

[24] D.F. James, R. Yip, I.G. Currie, Slow flow of Boger fluids through model fibrous porous media, J. Rheol. 56 (2012) 1249-1277.

[25] J. Avendano, N. Pannacci, B. Herzhaft, P. Gateau, P. Coussot, Enhanced displacement of a liquid pushed by a viscoelastic fluid in a straight channel, submitted, 2013.

[26] D.V. Boger, A highly elastic constant-viscosity fluid, J. Non-Newton. Fluid Mech. 3 (1977) 87-91.

[27] V. Gauri, K.W. Koelling, Extensional rheology of concentrated poly(ethylene oxide) solutions, Rheol. Acta. 36 (1997) 555-567.

[28] A. Ait-Kadi, P.J. Carreau, G. Chauveteau, Rheological Properties of Partially Hydrolyzed Polyacrylamide Solutions, J. Rheol. 31 (1987).

[29] J. Stokes, L. Graham, N. Lawson, D. Boger, Swirling flow of viscoelastic fluids. Part 2. Elastic effects, J. Fluid Mech. 429 (2001) 117-153.

[30] C.J. Pipe, G.H. McKinley, Microfluidic rheometry, Mech. Res. Commun. 36 (2009) 110-120.

[31] D.M. Jones, K. Walters, P.R. Williams, On the extensional viscosity of mobile polymer solutions, Rheol. Acta. 26 (1987) 20-30.

[32] P.C. Sousa, P.M. Coelho, M.S.N. Oliveira, M.A. Alves, Three-dimensional flow of Newtonian and Boger fluids in square-square contractions, J. Non-Newtonian Fluid Mech., 160 (2009) 122-139.

[33] D.J. Tritton, Physical Fluid Dynamics, Clarendon Press, 1988. 